\title{
The Clinical Implications of Overlap Between Constipation and Common Functional Gastrointestinal Disorders
}

\author{
Kang Nyeong Lee \\ Department of Internal Medicine, Hanyang University College of Medicine, Seoul, Korea
}

\begin{abstract}
Article: Nationwide multicenter study for overlaps of common functional gastrointestinal disorders in Korean patients with constipation

Park KS, Jee SR, Lee BE, et al

(J Neurogastroenterol Motil 2017;23:569-577)
\end{abstract}

Functional gastrointestinal disorders (FGIDs) are classified and categorized by the Rome criteria based on patients' symptoms, but some FGIDs have common pathophysiologic mechanisms, such as visceral hypersensitivity, dysmotility, altered interactions of gut and brain, dysregulated mucosal immunity, and so on. Probably because of these shared pathophysiologic mechanisms, patients with one FGID may also have other FGIDs, or complain of the corresponding symptoms. Such overlap of FGIDs has been reported between functional dyspepsia (FD), gastroesophageal reflux disease (GERD), and irritable bowel syndrome (IBS). ${ }^{1}$ Also, non-gastrointestinal (GI) diseases, including functional somatic syndromes such as fibromyalgia and overactive bladder syndrome, can overlap with FGIDs. The most frequent overlap has been reported between FD and IBS: studies have shown that $32 \%$ of patients with FD also have IBS, and up to $37 \%$ of IBS patients also have FD., ${ }^{2,3}$ A Korean study has also reported an approximately $50 \%$ overlap between FD and IBS. ${ }^{4}$

However, whether constipation overlaps with common FGIDs such as FD and GERD has been infrequently investigated. In this edition of the Journal of Neurogastroenterology and Motility, the authors publish a prospective, nationwide multicenter study using validated questionnaires including quality of life (QOL). ${ }^{5}$ In their study, the authors report the prevalence of overlap between constipation and FD or GERD, risk factors for such overlap, and the influence of overlap on QOL. Of the 759 study subjects with constipation enrolled from 19 centers (59.4\% of functional constipation and $40.6 \%$ of constipation-predominant IBS [IBS-C]) $17.9 \%$ also had GERD, 10.5\% had FD, and 6.7\% had both GERD and FD. On the other hand, 492 (64.8\%) of the 759 subjects did not have GERD or FD. Overlap with GERD or FD was more frequent in those with IBS-C than in those with functional constipation. The authors also show that overlap was associated with such factors as laxatives use, low intake of fiber, pulmonary diseases, and herniated nucleus pulposus. Constipated patients with overlaps with other FGIDs also showed more severe symptoms, and constipation-associated or general QOL was poorer than those without. Although the authors did not subdivide FD and GERD-dividing FD into epigastric pain syndrome and postprandial distress syndrome, and GERD into erosive esophagitis, non-erosive reflux disease, reflux hypersensitivity, and functional heartburn, a previous study has re-

Received: September 17, 2017 Revised: None Accepted: September 22, 2017

(.) This is an Open Access article distributed under the terms of the Creative Commons Attribution Non-Commercial License (http://creativecommons. org/licenses/by-nc/4.0) which permits unrestricted non-commercial use, distribution, and reproduction in any medium, provided the original work is properly cited.

${ }^{*}$ Correspondence: Kang Nyeong Lee, MD, PhD Department of Internal Medicine, Hanyang University College of Medicine, 222 Wangsimni-ro, Seongdong-gu, Seoul 04763, Korea Tel: +82-2-2290-8339, Fax: +82-2-2290-8314, E-mail: leekn@hanyang.ac.kr 
ported that postprandial distress syndrome patients frequently suffer from IBS-C. ${ }^{6}$

The question whether the various manifestations of constipated patients define a single disease entity, or rather are shared symptoms of two or more types of FGID, has not been clearly answered. Regardless, in managing patients with constipation, and with other common FGIDs as well, overlap syndromes between constipation and GERD or FD should be recognized and considered. As shown by this study, patients with overlap syndromes tend to complain of severer symptoms ${ }^{7}$ as well as psychological comorbidities ${ }^{8}$ and poorer QOL. ${ }^{9}$ Although there is no consensus on the optimal management of patients with overlap syndromes, appropriate medications, such as motility modulators and pain modulators, should be selected. ${ }^{10,11}$ Importantly, management of patients with such overlaps should also be focused on psychological factors influencing QOL, because their responses to general treatments are likely to be poorer than those of patients with constipation alone.

\section{Financial support: None.}

\section{Conflicts of interest: None.}

\section{References}

1. Fujiwara Y, Arakawa T. Overlap in patients with dyspepsia/functional dyspepsia. J Neurogastroenterol Motil 2014;20:447-457.

2. Suzuki H, Hibi T. Overlap syndrome of functional dyspepsia and irri- table bowel syndrome - are both diseases mutually exclusive? J Neurogastroenterol Motil 2011;17:360-365.

3. Nakajima S, Takahashi K, Sato J, et al. Spectra of functional gastrointestinal disorders diagnosed by Rome III integrative questionnaire in a Japanese outpatient office and the impact of overlapping. J Gastroenterol Hepatol 2010;25(suppl 1):S138-S143.

4. Park JM, Choi MG, Cho YK, et al. Functional gastrointestinal disorders diagnosed by Rome III questionnaire in Korea. J Neurogastroenterol Motil 2011;17:279-286.

5. Park KS, Jee SR, Lee BE, et al. Nationwide multicenter study for overlaps of common functional gastrointestinal disorders in Korean patients with constipation. J Neurogastroenterol Motil 2017;23:569-577.

6. Talley NJ, Dennis EH, Schettler-Duncan VA, Lacy BE, Olden KW, Crowell MD. Overlapping upper and lower gastrointestinal symptoms in irritable bowel syndrome patients with constipation or diarrhea. Am J Gastroenterol 2003;98:2454-2459.

7. Corsetti M, Caenepeel P, Fischler B, Janssens J, Tack J. Impact of coexisting irritable bowel syndrome on symptoms and pathophysiological mechanisms in functional dyspepsia. Am J Gastroenterol 2004;99:11521159.

8. Piacentino D, Cantarini R, Alfonsi M, et al. Psychopathological features of irritable bowel syndrome patients with and without functional dyspepsia: a cross sectional study. BMC Gastroenterol 2011;11:94.

9. Kaji M, Fujiwara Y, Shiba M, et al. Prevalence of overlaps between GERD, FD and IBS and impact on health-related quality of life. J Gastroenterol Hepatol 2010;25:1151-1156.

10. Drossman DA. Beyond tricyclics: new ideas for treating patients with painful and refractory functional gastrointestinal symptoms. Am J Gastroenterol 2009;104:2897-2902.

11. Sperber AD, Drossman DA. Review article: the functional abdominal pain syndrome. Aliment Pharmacol Ther 2011;33:514-524. 